Introduction The return-to-work (RTW) process after breast cancer (BC) can be complex. Simple static measures used to assess RTW may ignore this dynamic multi-stage process that are yet important for targeting interventions aimed at reducing poor RTW outcomes.

Objectives Our aim was to identify RTW trajectories after BC using the sequence analysis method and describe their underpinning personal, medical, psychological and occupational factors.

Methods This study is based on the ELCCA II cohort (Angers, France) that recruited 128 women diagnosed with BC between February 2015 and April 2016. Among them, 96 were still participating at the four-year follow-up. An occupational calendar was used to assess their situation every 6 months from their diagnostic up to 4 years later. Women that did not complete their occupational calendar, that were inactive (retired, disability pension) at the time of their diagnosisic were excluded leading to a final sample of 52 breast cancer survivors (BCS). Sequence analysis was used to identify RTW trajectories among BCS. Patterns of RTW trajectories were described by socio-demographic, medical, occupational and the functional scales of the EORTC-QLQ-C30.

Results Three types of RTW trajectories were identified among BCS by sequence analysis: long sickness absence with progressive RTW $(n=18)$, short sickness absence and full time RTW ( $\leq 6$ months) $(n=27)$, short sickness absence with part-time RTW $(n=7)$. Socio-demographic, medical and trend in functional scales during the first year after diagnosis were shown to vary according RTW trajectories.

Conclusion The application of sequence analysis highlights the dynamic process of RTW among BCS. It captures trajectories of multiple states and transitions that provided a holistic and diachronic approach of RTW. Three different patterns of RTW trajectories after breast cancer were identified. However, these results have to be confirmed by using a larger sample of BCS.

\section{P-286 AN INVENTORY OF OCCUPATIONAL CANCER STUDIES AND THEIR EXPOSURE ASSESSMENT METHODS IN IRAN}

'Bayan Hosseini, Amy Hall, Kazem Zendehdel, Hans Kromhout, Felix Onyije, Rahmatollah Moradzadeh, Maryam Zamanian, Joachim Schuz, Ann Olsson. 'International Agency for Research on Cancer, France

\subsection{6/OEM-2021-EPI.262}

Introduction The presence of occupational carcinogens in workplaces in Iran are not well characterized, and little is known about the burden of occupational cancer in Iran and other Middle East countries.

Objectives This study aimed to provide a comprehensive overview of occupational cancer studies in Iran and describe how exposures were assessed and assigned in those studies.

Methods Studies addressing exposure to and health effects from carcinogens in Iran until the end of 2020 were identified through three databases (PubMed, Web of Science, and Google Scholar). Two reviewers independently screened the articles according to the PRISMA (Preferred Reporting Items for Systematic Reviews and Meta-Analyses) guideline. Data extracted from each eligible paper included geographical region, study design, occupation, industry, exposure agents, population size/sample size, exposure assessment methods, and cancer sites.
Results Forty-nine publications from inception to 2020 (1 cohort, 11 case-control, 34 exposure monitoring studies, and 3 other) were included. The case-control studies used selfreported job titles and/or self-reported exposures to estimate the associated risk of cancer, while exposure monitoring studies applied exposure measurements (including personal and stationary air sampling).

Conclusion Occupational cancer epidemiology in Iran is at an early stage, as evidenced by relatively small number of published articles on this topic. A coherent strategy to assess occupational exposures and cancer burden in Iran should include systematic inventory and monitoring of exposure to carcinogens for use in hazard control and research, as well as targeted tools and studies to assess unique occupational environments and their potential associated cancer hazards.

\section{P-290 NUMBER OF JOBS, JOBS DURATION AND RISK OF PROSTATE CANCER: A CASE-CONTROL STUDY IN MONTREAL, CANADA}

${ }^{1}$ Charlotte Salmon, Marie-Elise Parent. 'Institut national de la recherche scientifique, Université du Québec, Canada

\subsection{6/OEM-2021-EPI.263}

Introduction Successive job changes may reflect precarious employment, job dissatisfaction or promotions, all of which can result in accrued stress, disrupted social connections and health-altering circumstances and behaviours. Evidence is lacking on the potential role of job stability in cancer development.

Objectives We investigated the association between the number of jobs held and jobs duration over the career, and the risk of prostate cancer, overall and by cancer aggressiveness.

Methods Data from the Prostate Cancer \& Environment Study, a population-based case-control study conducted in 2005-2012 in Montreal, Canada were used. Incident cases $(n=1,931)$, aged $<76$ years, were ascertained across hospitals in the Montreal area. Population controls $(n=1,994)$ from the same area, frequency-matched to cases by age ( \pm 5 years), were identified from the electoral list. Information on the number of paid jobs held for $\geq 1$ year throughout the career and the duration of each job was elicited during face-to-face interviews. Unconditional logistic regression was used to assess odds ratios (OR) and 95\% confidence intervals (CI), adjusting for age, ancestry and education.

Results Subjects had held 5 jobs, on average ( \pm 3 ), with a mean job duration of 11.1 years $( \pm 7.9)$. A greater number of jobs held was associated with a higher risk of prostate cancer. The OR for men who had had $>6$ jobs was $1.23(95 \%$ CI $=1.04-1.46$ ), compared to men who had held $1-3$ jobs, with no differences according to cancer aggressiveness. Conversely, men who had held shorter jobs, on average, had a higher risk of prostate cancer, following a dose-response pattern (p-trend 0.047). The association was more pronounced for non-aggressive tumours.

Conclusion Our results suggest that job stability throughout the career may have a protective effect against prostate cancer. Further in-depth analyses are warranted. These findings are novel and require replication. 